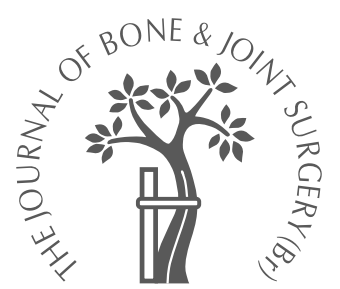

H. Katagiri,

M. Takahashi,

K. Wakai,

H. Sugiura,

T. Kataoka,

K. Nakanishi

From Shizuoka

Cancer Center

Hospital, Shizuoka,

Japan

- H. Katagiri, MD, PhD, Senior

Staff, Orthopaedic Surgeon

M. Takahashi, MD, PhD

Chief, Orthopaedic Surgeon

Division of Orthopaedic

Oncology, Shizuoka Cancer

Center Hospital, 1007

Shimonagakubo,

Nagaizumicho, Sunto-gun,

Shizuoka 411-8777, Japan.

- K. Wakai, MD, PhD, Section Head, Epidemiologist

Division of Epidemiology and

Prevention

H. Sugiura, MD, PhD, Chief,

Orthopaedic Surgeon

Division of Orthopaedic

Oncology

Aichi Cancer Center, 1-1

Kanokoden, Chikusa-ku,

Nagoya-City 464-8681, Japan.

- T. Kataoka, MD, PhD, Chief, Clinical Oncologist

Division of Clinical Oncology

Nagoya Memorial Hospital, 4-

305 Hirabari, Tenpaku-ku,

Nagoya-City 468-8520, Japan.

K. Nakanishi, MD, PhD,

Chief, Orthopaedic Surgeon

Division of Orthopaedic

Surgery, Prefectural Aich

Hospital, Kake-machi,

Okazaki-City 444-0011, Japan.

Correspondence should be sent to $\mathrm{Dr} \mathrm{H}$. Katagiri; e-mail: h.katagiri@scchr.jp

(C)2005 British Editorial

Society of Bone and

Joint Surgery

doi:10.1302/0301-620X.87B5. $15185 \$ 2.00$

$J$ Bone Joint Surg [Br] 2005;87-B:698-703.

Received 2 December 2003

Accepted after revision

8 October 2004

\title{
Prognostic factors and a scoring system for patients with skeletal metastasis
}

\begin{abstract}
Between 1992 and 1999, we treated 350 patients with skeletal metastases. A multivariable analysis of the patients was conducted using the Cox proportional hazards model. We identified five significant prognostic factors for survival, namely, the site of the primary lesion, the performance status (Eastern Cooperative Oncology Group status 3 or 4), the presence of visceral or cerebral metastases, any previous chemotherapy, and multiple skeletal metastases. The score for each significant factor was derived from the corresponding estimated regression coefficients (natural logarithm of the hazard ratio). The prognostic score was calculated by adding all the scores for individual factors.

The rate of survival was $31 \%$ at six months and $11 \%$ at one year for the patients with a prognostic score of $\mathbf{6}$ or more. By contrast, patients with a prognostic score of 2 or less had a rate of survival of $98 \%$ at six months and $89 \%$ at one year. This scoring system can be used to determine the optimal treatment for patients with pathological fractures or epidural compression.
\end{abstract}

For most patients with metastatic bone tumours, the aim of treatment is not to cure the disease but to improve mobility and the quality of life during their remaining weeks or months. There are a variety of options for the treatment of bone metastases. For the spine some patients are treated non-surgically by radiotherapy and/ or chemotherapy. Others have posterior decompression with or without instrumentation, and some an anterior excisional procedure. Tomita et $\mathrm{al}^{1}$ reported a more radical operation which entailed removing the lesion en bloc (spondylectomy). For metastases to bones in the limbs, external fixation, internal fixation without resection, or wide resection followed by reconstruction with a prosthesis is chosen depending on the condition of the patient.

When deciding treatment, the surgeon must take into consideration the degree of functional impairment, the general condition of the patient and his or her life expectancy. It is often difficult to predict the prognosis with any accuracy. In 1990, Tokuhashi et al $^{2}$ described a scoring system for the pre-operative evaluation of survival after surgery of a metastatic spinal tumour. Recently, Tomita et $\mathrm{al}^{3}$ have described a surgical strategy for spinal metastases. However, since these studies were retrospective and included only surgically-treated patients, few critical conclusions can be drawn as to their effectiveness.
In this prospective study, we sought to determine prognostic factors from the clinical features which can be obtained at presentation, and to create a prognostic model for patients with bone metastases.

\section{Patients and Methods}

Our study group was comprised of 350 consecutive patients who had undergone treatment, either surgical or non-surgical, for skeletal metastases between July 1992 and December 1999. The last follow-up evaluation of the patients was performed in January 2002. There were 199 men and 151 women with a mean age of 59 years ( 14 to 88 ).

Of the 350 patients, 326 (93\%) were followed for a minimum of 24 months, unless death supervened, during which time 24 patients were lost to follow-up. They were treated as 'censored observations'. Two deaths from causes other than malignancy were also treated as censored observations. The mean follow-up periods were 13 months (2 weeks to 88 months) for patients dying from their malignant disease, and 39 months (1 to 142) for survivors. Multiple myeloma, requiring orthopaedic care was treated as skeletal metastasis. ${ }^{4-8}$ However, solitary plasmacytoma was excluded from the study as was malignant lymphoma of bone without an extraosseous lesion. Patients with a pri- 
Table I. The primary tumours in the 350 patients

\begin{tabular}{lc}
\hline Primary lesion & Number of patients \\
\hline Lung carcinoma & 82 \\
Breast carcinoma & 64 \\
Prostate carcinoma & 28 \\
Multiple myeloma & 24 \\
Hepatocellular carcinoma & 21 \\
Gastric carcinoma & 20 \\
Malignant lymphoma & 19 \\
Colon and rectal carcinoma & 16 \\
Renal-cell carcinoma & 16 \\
Bone and soft-tissue sarcoma & 16 \\
Thyroid carcinoma & 9 \\
Endometrial, cervical and ovarian carcinoma & 9 \\
Gall-bladder and bile-duct carcinoma & 7 \\
Double carcinoma & 2 \\
Other & 9 \\
Unknown origin & 8 \\
Total & 350
\end{tabular}

mary lymph-node lesion and secondary bone metastases were included.

Lung carcinoma was the most common primary lesion. Others were carcinoma of the breast, prostate, liver and multiple myeloma (Table I). The primary lesion was not found in eight patients despite thorough investigation.

Treatment. Of the 350 patients, $249(71 \%)$ were treated non-surgically while the remaining 101 had an operation for skeletal metastasis. Of those treated without surgery, two were given palliative care alone, 27 were treated by chemotherapy including hormonal preparations, 94 had radiotherapy alone, and 126 had combined chemotherapy and radiotherapy. Of the 101 patients treated by surgery, internal fixation or endoprosthetic replacement was performed in 56, posterior decompression and instrumentation in 37 , endoprosthetic replacement and spinal instrumentation in one, resection without reconstruction in six, and amputation in one. Fifteen had surgery alone and the remaining 86 were given additional radiotherapy and/ or chemotherapy. All patients gave their informed consent for each examination and treatment.

Imaging study. Plain radiography of any obviously affected bone and whole-body bone scanning using $99^{\mathrm{m}} \mathrm{Tc}$ methylene diphosphonate (MDP) were carried out routinely in every patient to determine the spread of bone metastases. MRI was performed to detect skeletal metastasis more precisely in 165 patients. Visceral or cerebral metastases were assessed by CT.

Statistical analysis. Twelve potentially prognostic factors were investigated (Table II). Each was grouped in up to three categories for statistical analysis. Age was categorised into groups of younger than 60 years and 60 years or older. Neurological deficit was divided into two groups, either present or not present. Performance status was evaluated using the Eastern Cooperative Oncology Group (ECOG)

Table II. Distribution of potentially prognostic factors in the 350 patients

\begin{tabular}{|c|c|c|c|c|}
\hline \multirow[b]{2}{*}{ Patient-related factor } & \multirow{2}{*}{$\begin{array}{l}\text { Prognostic factors } \\
\text { Gender }\end{array}$} & \multirow{2}{*}{$\begin{array}{l}\text { Subgroups } \\
\text { Male }\end{array}$} & \multicolumn{2}{|c|}{$\begin{array}{l}\text { Number of } \\
\text { patients }(\%)\end{array}$} \\
\hline & & & 199 & 57 \\
\hline & & Female & 151 & 43 \\
\hline & Age (yrs) & $<60$ & 140 & 40 \\
\hline & & $\geq 60$ & 210 & 60 \\
\hline & Performance status & PS* 0 to 2 & 131 & 37 \\
\hline & & PS 3 to 4 & 219 & 63 \\
\hline & Neurological deficits $†$ & No & 248 & 71 \\
\hline & & Yes & 101 & 29 \\
\hline \multirow[t]{8}{*}{ Primary-site-related factor } & Primary lesion & $\begin{array}{l}\text { Carcinoma of the breast, prostate, thyroid, multiple } \\
\text { myeloma and malignant lymphoma }\end{array}$ & 144 & 41 \\
\hline & & $\mathrm{HCC} \neq$, carcinoma of the stomach or lung & 132 & 38 \\
\hline & & Other carcinomata and sarcoma & 74 & 21 \\
\hline & Remaining primary disease & No & 125 & 36 \\
\hline & & Yes & 225 & 64 \\
\hline & & Yes & 178 & 51 \\
\hline & Previous chemotherapy $\dagger$ & No & 241 & 69 \\
\hline & & Yes & 108 & 31 \\
\hline \multirow[t]{9}{*}{ Skeletal-metastasis-related factor } & Diseased-free interval & No & 160 & 46 \\
\hline & & Yes & 190 & 54 \\
\hline & Location of skeletal metastases & Appendicular bone & 41 & 12 \\
\hline & & Axial bone & 222 & 63 \\
\hline & & Appendicular and axial bone & 87 & 25 \\
\hline & Multiple metastases & No & 112 & 32 \\
\hline & & Yes & 238 & 68 \\
\hline & Pathological fracture $†$ & No & 191 & 55 \\
\hline & & Yes & 158 & 45 \\
\hline
\end{tabular}

* PS, performance status

$\dagger$ in one patient, information was not available

₹ HCC, hepatocellular carcinoma 


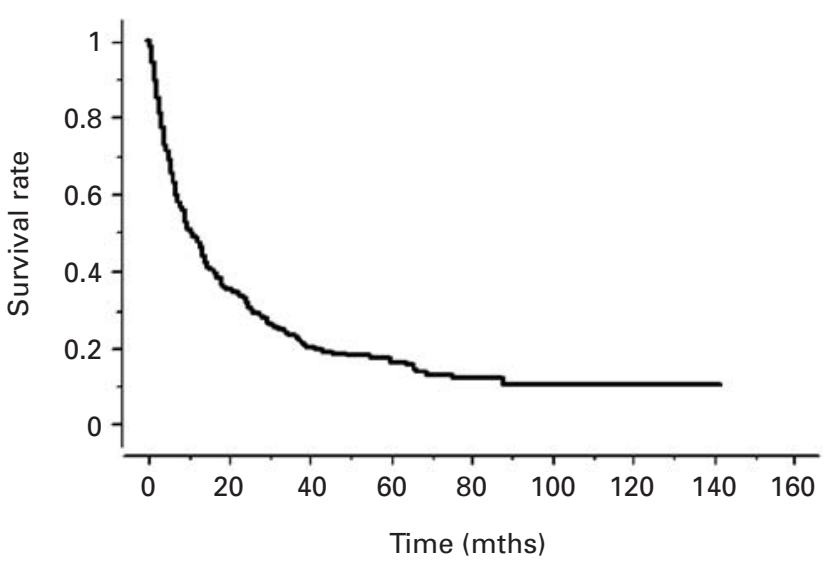

Fig. 1

Kaplan-Meier cumulative survival rate for the 350 patients.

performance status scale ${ }^{9}$ and categorised into two groups of performance status 0 to 2 and 3 to 4 . Primary sites of cancer were classified into three groups. Lung, gastric and hepatocellular carcinoma were categorised as a rapid growth group, breast and prostate carcinoma, multiple myeloma, malignant lymphoma, and thyroid carcinoma as a slow growth group; and other carcinomas and sarcomas as a moderate growth group. The condition of the primary lesion was divided into two categories according to whether it was already cured or untreated and recurring. Previous chemotherapy was divided into two categories, no previous chemotherapy and any previous therapy including hormonal treatment and intra-arterial infusion chemotherapy.
Visceral or cerebral metastases were grouped into two categories, no visceral or cerebral metastasis and any detectable by imaging. The disease-free interval from primary cancer to the development of skeletal metastases was divided into two categories, no disease-free interval in which skeletal metastases were evident on presentation and a disease-free interval during which skeletal metastases developed after treatment of the primary lesion. The sites of skeletal metastases were divided into three categories, limited to appendicular bone, limited to axial bone and present in both. The skeletal metastatic load was subdivided into two categories, solitary or multiple. Pathological fractures were also divided into two categories, present or not on imaging.

Rates of survival were calculated by the Kaplan-Meier method..$^{10}$ All of the factors shown in Table II were included as explanatory variables in a Cox proportional-hazards survival analysis. ${ }^{11}$ The results of the multivariate analyses were expressed in terms of hazard ratio derived from the estimated regression coefficients, with $95 \%$ confidence intervals (CI). The hazard ratio indicates how many times patients with each factor are more likely to die compared with those without the factor or in the reference group. In this analysis, categorical variables with more than two modalities were recorded using dummy variables. We used the SAS statistical package (SAS, Chicago, Illinois).

The score for each significant prognostic factor was derived from the corresponding estimated regression coefficients (natural logarithm of the hazard ratio) of the multivariate model. The corresponding estimated regression coefficients were multiplied by two and rounded off to the nearest integer in order to make the calculation of the prognostic score as simple as possible by allocating one point for

Table III. Multivariate analysis for prognostic factors

\begin{tabular}{|c|c|c|c|c|c|}
\hline Variable (coding) & $\begin{array}{l}\text { Regression } \\
\text { coefficient }\end{array}$ & Standard error & Test statistic & $\mathrm{p}$ value & Hazard ratio $(95 \% \mathrm{Cl}) *$ \\
\hline Gender (female:0, male:1) & -0.115 & 0.144 & 0.641 & 0.423 & 0.891 (0.672 to 1.181$)$ \\
\hline Age in years $(>60: 0, \leq 60: 1)$ & 0.048 & 0.139 & 0.122 & 0.727 & $1.050(0.800$ to 1.378$)$ \\
\hline PSt (PS 0 to 2:0, PS 3 to $4: 1$ ) & 0.402 & 0.151 & 7.112 & 0.008 & 1.495 (1.112 to 2.008$)$ \\
\hline Neurological deficits $\ddagger$ & 0.009 & 0.157 & 0.004 & 0.953 & 1.009 (0.743 to 1.372$)$ \\
\hline \multicolumn{6}{|l|}{ Primary site§ } \\
\hline 2 & 0.908 & 0.193 & 22.142 & $<0.001$ & 2.479 (1.699 to 3.619$)$ \\
\hline 3 & 1.646 & 0.177 & 86.109 & $<0.001$ & 5.189 (3.665 to 7.346$)$ \\
\hline Remaining primary disease $\ddagger$ & 0.265 & 0.189 & 1.974 & 0.160 & $1.304(0.901$ to 1.887$)$ \\
\hline Visceral or cerebral metastasesł & 0.796 & 0.136 & 34.075 & $<0.001$ & 2.217 (1.697 to 2.897 ) \\
\hline Previous chemotherapy $\ddagger$ & 0.431 & 0.180 & 5.711 & 0.017 & 1.538 (1.081 to 2.190$)$ \\
\hline \multirow{2}{*}{\multicolumn{6}{|c|}{$\begin{array}{l}\text { Location of skeletal metastases } \\
\text { Appendicular bone (reference group) }\end{array}$}} \\
\hline & & & & & \\
\hline Axial bone & 0.303 & 0.248 & 1.500 & 0.221 & 1.354 (0.834 to 2.200$)$ \\
\hline Appendicular and axial bone & 0.186 & 0.278 & 0.447 & 0.504 & 1.204 (0.698 to 2.078 ) \\
\hline Multiple skeletal metastasis $¥$ & 0.339 & 0.171 & 3.927 & 0.048 & 1.404 (1.004 to 1.964$)$ \\
\hline Pathological fracture $\ddagger$ & 0.144 & 0.136 & 1.127 & 0.288 & 1.155 (0.885 to 1.508$)$ \\
\hline
\end{tabular}


Table IV. Significant prognostic factors and score for each factor

\begin{tabular}{llc}
\hline Prognostic factor & & Score \\
\hline $\begin{array}{ll}\text { Primary lesion } \\
\quad \text { Rapid growth }\end{array}$ & Hepatocellular carcinoma, gastric carcinoma, lung carcinoma & 3 \\
\multicolumn{1}{c}{ Slow growth } & Breast carcinoma, prostate carcinoma, multiple myeloma, & 0 \\
\multicolumn{1}{l}{ Moderate growth } & Malignant lymphoma, thyroid carcinoma & 2 \\
Visceral or cerebral metastases & Other carcinoma and sarcoma & 2 \\
Performance status (ECOG)* 3 or 4 & & 1 \\
Previous chemotherapy & & 1 \\
Multiple skeletal metastases & & 1 \\
\hline
\end{tabular}

* ECOG, Eastern Cooperative Oncology Group ${ }^{9}$

factors with the smallest regression coefficients. The scoring system was more accurate if the estimated regression coefficients were multiplied by a larger number. However, we gave priority to the simplicity of the scoring system in order to facilitate its use in clinical practice. The prognostic score was calculated by adding all the scores for individual factors. Every patients was scored from 0 to 8 , and divided into nine groups according to the prognostic score. The rates of survival for each group were calculated using the Kaplan-Meier method.

\section{Results}

The overall rate of survival of the entire group was 0.66 at six months, 0.48 at 12 months, 0.33 at 24 months and 0.23 at 36 months (Fig. 1). With multivariate analysis, the site of the primary tumour, ECOG performance status 3 or 4, visceral or cerebral metastases, previous chemotherapy and multiple skeletal metastases were found to be significant, independent, prognostic factors (Table III).

The highest hazard ratios were found for the primary site of the tumour. The results indicate that the patients with a group 3 primary site (gastric, lung and hepatocellular carcinoma) and those with a group 2 primary site (carcinoma and sarcoma other than those in groups 1 and 3) were 5.19 and 2.48 times, respectively, more likely to die than those with a group 1 primary site (breast, prostate and thyroid carcinoma, multiple myeloma and malignant lymphoma). Patients with visceral or cerebral metastases were at a 2.22fold risk of death compared with those without such lesions.

The hazard ratios for performance status, previous chemotherapy, and multiple skeletal metastases were 1.50 , 1.54 and 1.40 , respectively. Patients with performance status 3 or 4 had a $50 \%$ higher risk of dying than those with more favourable performance status. Patients who had had previous chemotherapy had a $54 \%$ higher risk and those with multiple skeletal metastases a $40 \%$ higher risk of dying than those without the factors.

The score for each significant prognostic factor derived from the corresponding estimated regression coefficients is shown in Table IV. The prognostic score was calculated by adding the scores for each significant prognostic factor for every patient. The Kaplan-Meier survival curves for different prognostic scores are shown in Figure 2. At 12 months,

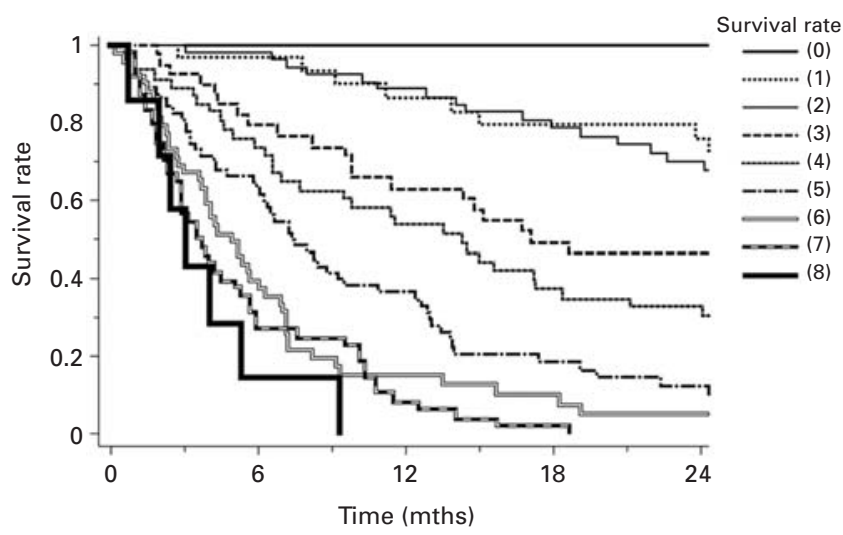

Fig. 2

Kaplan-Meier survival curves for different prognostic scores. Values in parentheses indicate the prognostic score.

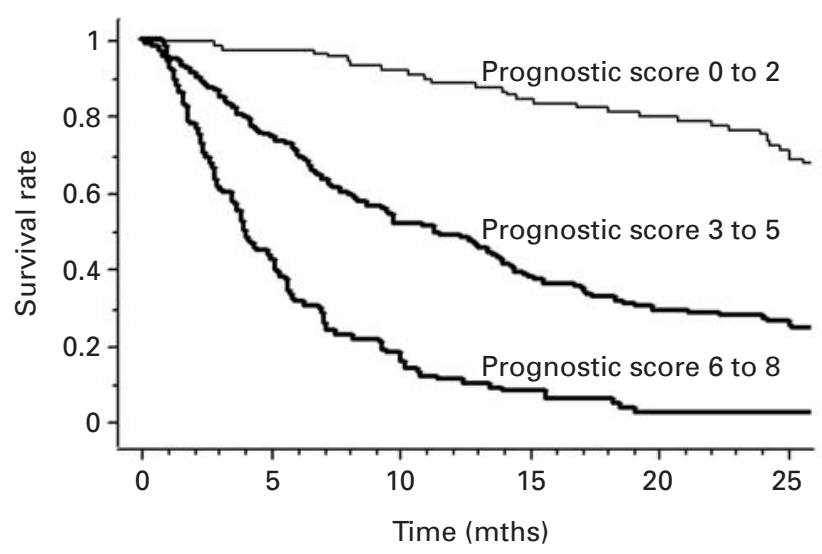

Fig. 3

Kaplan-Meier survival curves of patients with prognostic scores of 0 to 2 , 3 to 5 , and 6 to 8 . The rates of survival for these three groups are significantly different (log-rank test, $p<0.0001$ )

the survival curves can be separated into three groups, namely, a score of 0 to 2 with a survival rate of more than $80 \%, 3$ to 5 with a survival rate of $30 \%$ to $70 \%$, and 6 to 8 with a survival rate of less than $20 \%$. The survival curves of these three groups are shown in Figure 3, and were sig- 
Table V. Prognostic score and survival rate (95\% confidence interval) at six, 12 and 24 months

\begin{tabular}{llll}
\hline & \multicolumn{3}{l}{ Survival rate (mths) } \\
\cline { 2 - 4 } Prognostic score & $\mathbf{6}$ & $\mathbf{1 2}$ & $\mathbf{2 4}$ \\
\hline 0 to 2 & $0.979(0.950$ to 1.000$)$ & $0.891(0.828$ to 0.955$)$ & $0.753(0.663$ to 0.843$)$ \\
3 to 5 & $0.706(0.633$ to 0.780$)$ & $0.488(0.406$ to 0.570$)$ & $0.278(0.202$ to 0.353$)$ \\
6 to 8 & $0.313(0.224$ to 0.401$)$ & $0.109(0.049$ to 0.169$)$ & $0.023(0.000$ to 0.053$)$ \\
\hline
\end{tabular}

nificantly different (log-rank test, $\mathrm{p}<0.0001)$. The rate of survival of patients with prognostic scores of 0 to 2,3 to 5 , and 6 to 8 at six months are $0.98,0.71$, and 0.31 , respectively, against $0.89,0.49$, and 0.11 , respectively at 12 months (Table V).

\section{Discussion}

There have been several reports on the prognosis for patients with skeletal metastases. Tokuhashi et $\mathrm{al}^{2}$ proposed a prognostic scoring system for the pre-operative evaluation of patients with spinal metastases comprising six variables: the Karnofsky performance status, the number of extraspinal bone metastases, the number of metastases in the vertebral body, metastases in the major organs, the primary site of cancer, and palsy of the spinal cord. Each variable was evaluated with a score from 0 to 2 points, giving a maximum total score of $12 .^{2}$ Bauer and Wedin ${ }^{4}$ reported five positive criteria for survival: absence of visceral metastases, absence of pathological fracture, solitary skeletal metastasis, a primary tumour of the breast or kidney and lymphoma or myeloma, but not lung cancer. Tomita et $\mathrm{al}^{3}$ reported a new scoring system for spinal metastases with three prognostic factors namely the primary tumour (slow, moderate or rapid growth), visceral metastases (treatable or untreatable), and bone metastases (solitary or isolated and multiple). The scoring systems of Tokuhashi et $\mathrm{al}^{2}$ and Tomita et $\mathrm{al}^{3}$ were based on retrospective analysis and the three studies analyse only surgicallytreated patients. Although our study was designed to include not only surgically but also non-surgically-treated patients, the primary tumour, visceral metastases, and multiple skeletal metastases were significant prognostic factors, as in the three earlier papers.

Teshima et $\mathrm{al}^{12}$ found that the primary site, extraskeletal metastases, performance status, age and the urinary hydroxyproline/creatinine ratio, were prognostic factors in patients treated by radiotherapy. The differences from our present study were that age was a significant prognostic factor, but multiple skeletal metastases were not. Their study was prospectively conducted on patients treated by radiotherapy and may have had a selection bias. The hydroxyproline/creatinine ratio was reported to be a significant prognostic factor but since it currently takes several days to obtain this value, routine use and feedback for the selection of therapeutic options are difficult.

It seems clear from our study that a neurological deficit and the location of a skeletal metastasis do not influence survival independently, but that previous chemotherapy is an independent prognostic factor. With regard to the neurological deficit at presentation, Tokuhashi et $\mathrm{al}^{2}$ considered it to be a parameter of the prognostic score, whereas Tomita et $\mathrm{al}^{3}$ presumed that a neurological deficit was not a factor. Neither study, however, included a statistical analysis. Speigel et $\mathrm{al}^{13}$ also reported that patients with a neurological deficit tended to have a short survival, but that this did not significantly influence survival among patients with melanoma. We found no evidence to show that a neurological deficit was a significant prognostic factor, which supports the supposition of Tomita et al. ${ }^{3}$

There has been little documentation regarding the location of skeletal metastases in the past, since most previous studies did not address this issue. Yamashita et $\mathrm{al}^{14,15}$ studied this in regard to prostate and breast carcinoma. They reported that a skeletal lesion limited to the lumbar spine and pelvis was a favourable prognostic factor among patients with skeletal metastases from prostate carcinoma, but skeletal metastases caudal to the lumbosacral junction were a negative prognostic factor in patients with bone metastases from breast carcinoma. ${ }^{14,15}$ From multivariate analysis, our three groupings according to site were not significantly associated with survival.

The finding that previous chemotherapy is a significant prognostic factor does not mean that chemotherapy itself makes survival worse. There could be two reasons for this. First, patients with advanced stage of the disease at the initial treatment of the primary lesion, tend to be given chemotherapy and secondly, those with previous chemotherapy have no or few effective options for chemotherapy left after skeletal metastases occur. Sherry et $\mathrm{a}^{16}$ reported that patients with breast cancer, who already had skeletal metastases at the time of presentation with the primary breast lesion, had a favourable prognosis, irrespective of multiple skeletal involvement detectable on bone scans. Therefore, we anticipated that the disease-free interval from primary cancer to the development of skeletal metastases might influence survival, but this was not found to be the case. Bauer and Wedin ${ }^{4}$ reported that pathological fracture was a negative prognostic factor. Skeletal metastases with higher malignancy are reported to grow invasively and destructively. ${ }^{17,18}$ Therefore, we anticipated that pathological fracture might be a negative prognostic factor, but this also was not found to influence survival.

There are many options for the treatment of bone metastases. For lesions of the limbs, surgery for apparent or impending fracture is attractive although sometimes it is difficult to choose between external fixation, internal fixa- 
tion, or excision followed by reconstruction. For spinal metastases, it is often difficult to decide between radiotherapy, posterior stabilisation and an anterior excisional procedure. Life expectancy of at least two months is usually required for surgery for metastases of the limbs, ${ }^{19,20}$ and of three to six months for surgery for those in the spine. .,21,22 $^{2}$

For long-term survivors, excisional surgery followed by reconstruction with an implant is preferred to achieve longlasting local control of a tumour and stabilisation. ${ }^{23,24} \mathrm{Sim}$ ilarly, an excisional procedure followed by instrumentation is preferred for long-term control of spinal metastases. ${ }^{2,3}$ For patients with a prognostic score of 0 to 2 , the expected rate of survival after one year is $89 \%$. Therefore, an excisional procedure followed by reconstruction by a cemented prosthesis is preferred for metastases of the limbs and excisional spinal surgery is preferred for spinal metastases if radiotherapy is not expected to be effective. For patients with a prognostic score of 3 to 5 , the expected rate of survival after six months is $71 \%$ and at one year $49 \%$. Therefore, an excisional procedure followed by reconstruction by a cemented prosthesis or internal fixation with augmentation by methylmethacrylate is preferred for metastases of the limbs and anterior or posterior instrumentation procedures in the spine if radiotherapy is not expected to be effective. By contrast, in patients with a prognostic score of 6 to 8 , the expected rate of survival after six months is $31 \%$ and at one year, $11 \%$. Simple internal fixation followed by radiotherapy is the first choice for patients with metastases of the limbs. These patients are not good candidates for spinal surgery and radiotherapy with supportive care is the treatment of choice for patients with spinal metastases.

When deciding upon treatment, we must consider not only the pain and degree of neurological impairment but also the sensitivity to chemotherapy and/or radiotherapy, the destructive spread of bone metastases and life expectancy. Patients with a very short life expectancy should probably be treated less invasively, and not all patients with a long life expectancy require aggressive surgery. With this practical prognostic scoring system, life expectancy may be predicted more accurately and thus the optimal treatment better selected.

This work was supported in part by the Grant-in-Aid for Cancer Research (1419) from the Ministry of Health, Labour and Welfare, Japan.

No benefits in any form have been received or will be received from a commercial party related directly or indirectly to the subject of this article.

\section{References}

1. Tomita K, Kawahara N, Baba H, et al. Total en bloc spondylectomy for solitary spinal metastases. Int Orthop 1994;18:291-8.

2. Tokuhashi Y, Matsuzaki H, Toriyama S, Kawano H, Ohsaka S. Scoring system for the preoperative evaluation of metastatic spine tumor prognosis. Spine 1990;15: 1110-13.

3. Tomita K, Kawahara N, Kobayashi T, et al. Surgical strategy for spinal metastases. Spine 2001;26:298-306.

4. Bauer HCF, Wedin R. Survival after surgery for spinal and extremity metastases. Acta Orthop Scand 1995;66:143-6.

5. Fidler MW. Anterior decompression and stabilization of metastatic spinal fractures. J Bone Joint Surg [Br] 1986;68-B:83-90.

6. Harrington KD. Anterior decompression and stabilization of the spine as a treatment for vertebral collapse and spinal cord compression from metastatic malignancy. Clin Orthop 1988;223:177-97.

7. DeWald RL, Bridwell KH, Prodromas C, Rodts M. Reconstructive spinal surgery as palliation for metastatic malignancies of the spine. Spine 1985;10:21-6.

8. Hosono N, Yonenobu K, Fuji T, et al. Vertebral body replacement with a ceramic prosthesis for metatastic spinal tumors. Spine 1995;20:2454-62.

9. Oken MM, Creech RH, Tormey DC, et al. Toxicity and response criteria of the Eastern Cooperative Oncology Group. Am J Clin Oncol 1982;5:649-55.

10. Kaplan EL, Meier P. Nonparametric estimation for incomplete observation. J Am Stat Assoc 1958; 53:457-81

11. Cox DR. Regression models and life tables. JR Stat Soc [B] 1972;34:187-220.

12. Teshima T, Chatani M, Inoue T, et al. Prognostic factors for patients with osseous metastasis: a multi-institutional prospective study. Strahlenther Onkol 1990;166: 387

13. Spiegel DA, Sampson JH, Richardson WJ, et al. Metastatic melanoma to the spine: demographics, risk factors, and prognosis in 114 patients. Spine 1995;20: 2141 6.

14. Yamashita K, Denno K, Ueda T, et al. Prognostic significance of bone metastases in patients with metastatic prostate cancer. Cancer 1993;71:1297-302

15. Yamashita K, Ueda T, Komatsubara Y, et al. Breast cancer with bone-only metastases: visceral metastases-free rate in relation to anatomic distribution of bone metastases. Cancer 1991:68:634-7.

16. Sherry MM, Greco FA, Johnson DH, Hainsworth JD. Breast cancer with skeleta metastases at initial diagnosis: distinctive clinical characteristics and favourable prognosis. Cancer 1986;58:178-82.

17. Milch RA, Changus GW. Response of bone to tumor invasion. Cancer 1956;9: 341-51.

18. Lodwick GS, Wilson AJ, Farrell C, Virtama P, Dittrich F. Determining growth rates of focal lesions of bone from radiographs. Radiology 1980;134:577-83.

19. Harrington KD. Orthopaedic management of metastatic bone disease. St. Louis, etc: The C.V. Mosby Company, 1988:141-3.

20. Harrington KD, Sim FH, Enis JE, et al. Methylmethacrylate as an adjunct in internal fixation of pathological fractures: experience with three hundred and seventy-five cases. J Bone Joint Surg [Am] 1976;58-A:1047-55.

21. Cybulski GR, Von Roenn KA, D'Angelo CM, Dewald RL. Luque rod stabilization for metastatic disease of the spine. Surg Neurol 1987;28:277-83.

22. Atanasiu JP, Badatcheff F, Pidhorz. Metastatic lesions of the cervical spine: a retrospective analysis of 20 cases. Spine 1993;18:1279-84.

23. Yazawa Y, Frassica FJ, Chao EY, et al. Metastatic bone disease: a study of the surgical treatment of 166 pathologic humeral and femoral fractures. Clin Orthop 1990; 251:213-19.

24. Rompe JD, Eysel P, Hopf C, Heine J. Metastatic instability at the proximal end of the femur: comparison of endoprosthetic replacement and plate osteosynthesis. Arch Orthop Trauma Surg 1994;113:260-4. 